(C) 2016 IEEE. Personal use of this material is permitted. Permission from IEEE must be obtained for all other uses, in any current or future media, including reprinting/republishing this material for advertising or promotional purposes, creating new collective works, for resale or redistribution to servers or lists, or reuse of any copyrighted component of this work in other works. 


\title{
Short-term Energy Storage for Power Quality Improvement in Weak MV Grids with Distributed Renewable Generation
}

\author{
Francisco Díaz-González, Gerard Del-Rosario-Calaf, Francesc Girbau-Llistuella, \\ and Oriol Gomis-Bellmunt
}

\begin{abstract}
The intermittent and stochastic nature of renewable energy sources impose several challenges for the operation of electrical networks, and affect power quality levels as well. This paper addresses one of the issues associated to power quality in electrical networks: voltage flicker. The main contribution of the paper fall in the proposal of a methodology for addressing the location in the network and the sizing of an active filter for flicker filtering. The designed active filter is based on controllable and bidirectional power electronics. Also, it includes energy storage capability, so as to maximize flicker filtering effectiveness by performing both active and reactive power regulation.
\end{abstract}

\section{INTRODUCTION}

The increase in electricity prices, reduction of fossil fuels and rising concerns on GreenHouse Gas (GHG) emissions, have risen the need for integrating Renewable Energy Sources (RES) into the power grid, requesting for a more modernized bidirectional power grid. The objective is to deliver more reliable, economical and sustainable electricity to final users [1]. RES are usually power converter interfaced, and have intermittent unpredictable nature, so the concerns on stability makes necessary to assess the power quality of the smart grid. The power quality problem such as voltage distortion may affect the performance of some critical loads in the distribution system, so it has to be treated carefully finding new techniques for the compensation of potential instabilities.

One of the power quality issues around voltage distortion is the so-called flicker phenomena. According to the international standard IEC 61000-4-15 [2], flicker is defined as a subjective sensation of visual instability provoked by fast fluctuations in light stimulus. Flicker is associated to those voltage fluctuations ranged from 0.05 to $35 \mathrm{~Hz}$ and, from experimental analyses, reaches its maximum at $8.8 \mathrm{~Hz}$. Flicker, since associated to voltage distortions, reduces power quality in distribution electrical grids and this carries different

F. Díaz-González is with Centre d'Innovació Tecnològica en Convertidors Estàtics i Accionaments (CITCEA-UPC), Universitat Politècnica de Catalunya ETS d'Enginyeria Industrial de Barcelona, C. Avinguda Diagonal, 647, P1. 2 , 08028 Barcelona, Spain (e-mail: francisco.diaz-gonzalez@citcea.upc.edu).

G. Del-Rosario-Calaf is with IREC, Catalonia Institute for Energy Research

F. Girbau-Llistuella is with Centre d'Innovació Tecnològica en Convertidors Estàtics i Accionaments (CITCEA-UPC)

O. Gomis-Bellmunt is with Catalonia Institute for Energy Research (IREC) and also with Centre d'Innovació Tecnològica en Convertidors Estàtics i Accionaments (CITCEA-UPC)

This work has been supported by the Seventh Framework Programme for Research of the European Commission under the project Smart Rural Grid. Grant agreement: 619610 consequences at different levels and for different actors of the system. For instance, proper operation and useful life of sensible end user equipment may be influenced by these voltage fluctuations, motivating the installation of ancillary equipment for protection [3]. Also, to respect a threshold level in flicker emission is also a requirement for the commission of renewable-based power plants such as wind parks [4]. For people protection, flicker must also be diminished since it can cause periodic annoying luminance changes in lamps that can derive in headaches and even in impaired visual performance and epileptic seizures [5].

So towards power quality improvement in general, and flicker minimization in particular, several systems have been proposed in the literature and/or developed in industry. These refer, for instance, to the installation of FACTS systems in the network for voltage control through reactive power regulation [6]. Also, they address the application of short-term storages, as in [7]. Further, proposals are for tackling flicker emission directly in the point of connection of distributed renewable generation, by smoothing the outputted power profiles, as in [8].

This paper analyses power quality in MV weak electrical networks with distributed energy resources. In particular, analyses are focused on flicker phenomena provoked by the randomness of renewable generation. As a technical solution for these aspects, the paper proposes the use of a power electronics-based active filter. The most remarkable aspect of this active filter is that it is able to exchange reactive power (as common active filters like FACTS systems do), but it is also capable of exchanging active power, as it includes short-term storages (e.g. flywheels and / or ultracapacitors). The main contribution of the paper is the deployment of a simulationbased procedure to address the optimal location and sizing of the active filter in the power system.

\section{FLICKER: AFFECTING FACTORS AND MEASUREMENT}

Flicker magnitude is mostly dependent on four aspects: i) the rated voltage level of the grid; ii) the strength and characteristics of the electrical network, i.e. the short-circuit power and ratio; iii) the magnitude and dynamics of active power fluctuations; iv) and the magnitude and dynamics of reactive power fluctuations [8].

This way, the variability of the power generated by distributed renewable sources is one of the principal affecting factors for flicker emission. In the case of wind turbines, the cycling torque disturbances at the generator shaft due to the 
airflow deviation through the tower section of the turbine, greatly disturbs the spectrum of the power generated in a frequency range directly related to flicker [9]. In the case of photovoltaics, the relationship with flicker emission is not clear enough yet. In the views of the authors of the present paper, further work is needed to characterize PV plant output in a frequency domain, as in [10], to identify and weight the magnitude of the power components in the frequency range related to flicker emission. This need to research is also expressed in [11]. This report recommends to research regulation concepts for inverters for power system voltage control to mitigate flicker and faster voltage fluctuations caused by local PV fluctuations. This report ensures that voltage fluctuations caused by PV power ramps due to cloud transients can affect solar irradiance up to $15 \%$ per second.

In regard of flicker measurement, emission severity can be determined according to the international standard IEC 610004-15 [2]. This standard describes the so-called flickermeter device, which provides, amongst others, two principal flicker indices for power quality assessment: short and long term flicker severity, and they are calculated from the measurement and processing of voltage signal at the required connection point. Short-term flicker severity index $P_{s t}$ requires measuring the RMS voltage level for at least 10 minutes, while measuring for up to 2 hours is needed for determining long-term flicker index $P_{l t}$. Precise mathematics building up flickermeter are offered in detail in the standard. For practicality, Table I summarizes admissible threshold flicker emission levels for the connection of fluctuating installations to MV networks, according to [12]. As being relevant for the purposes of the present work, these are used as reference levels.

TABLE I. ADMISSIBLE LEVELS FOR SHORT AND LONG TERM FLICKER EMISSION ACCORDING TO IEC61000-3-7 STANDARD

\begin{tabular}{cc}
\hline$P_{s t}[-]$ & $P_{l t}[-]$ \\
\hline 0.35 & 0.25 \\
\hline
\end{tabular}

The above-presented affecting factors and flicker measurement techniques will serve to establish a design methodology for an active flicker filter system in the following section.

\section{DESIGN METHODOLOGY}

This section aims to establish a design methodology for an active filter to be utilized in electrical networks for power quality improvement (see the phases in Figure 1).

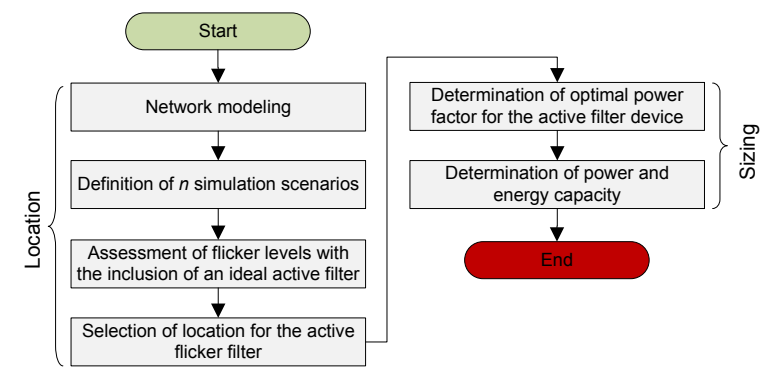

Fig. 1. Design methodology for the active filter.

\section{A. Location assessment}

The determination of the point of connection of the active filter in the power system is not trivial at all and as such it should be properly addressed. Bearing in mind an electrical network with distributed and renewable generation, one could come up with different criteria for the location of an active filter for flicker level minimization. Two main different criteria are identified here: $i$ ) The location of the active filter in the point of maximum flicker emission. This point would probably be at the connection point of a renewable generating power plant such as a wind park or stand alone wind turbine (depending on the size and topology of the network). The main advantage of doing this is that it will serve to facilitate the grid connection of the generating facility under consideration, for power quality standard compliance. On the other hand, this approach may not maximize the benefit the rest of the network actors could gain. This is because, for instance, critical loads or consumers could be connected far from the point of connection of the active flicker filter and still being affected by flicker emission provoked by other flicker sources located nearby; ii) The location of the active filter in a point so as to ensure a minimum threshold level of power quality throughout the network. Addressing the objective of the present paper, power quality is evaluated in terms of flicker phenomena.

The above introduced second criteria is adopted here, as it is considered more interesting than the first one from a power system engineering point of view. Adopting this criteria, the optimal location, $i^{*}$, for the active filter would be that minimizing the maximum registered short-term flicker indices $P_{s t \text { max }}$ for each of the buses of the network feeding loads $i$, while under the conditions defining all considered network scenarios $j$. This optimization criteria is translated into the following objective function

$$
z=\min _{\left(i^{*}, i, j\right)} P_{\text {st_max }}\left(i^{*}, i, j\right) .
$$

In this manner, the optimization criteria requires to explore all possible locations in the network for the active filter. Further, two important aspects while doing this are worth mentioning at this point: $i$ ) A set of simulation scenarios $j$ should be defined for exploration. These are characterized by considering different load and renewable generation levels. The number of analysis to be carried out can be diminished though, by identifying the worst case scenario; those scenarios presenting low flicker levels throughout the network can be left out of discussion; ii) The optimal location would be that presenting the highest sensitivity to filtering actions. To do that, an ideal flicker filter is considered at this point of development till addressing its sizing. This ideal device is to be presented in Section IV-C.

\section{B. Sizing of the active filter}

Once the location of the active filter is determined, the system is sized at this point of the design procedure. Sizing is intended here as the procedure for determining the energy storage capacity of the device to be able to exchange the required active power with the grid, as well as its rated apparent power, which is also derived from the reactive power needs for filtering purposes. 
The device could be operated so that it exchanges just active or reactive power for flicker filtering. However, the maximum effectiveness of the device in filtering is achieved by exchanging both active and reactive power at the same time. Thus, in this section the optimal power factor, $\cos \phi$, to be developed by the device for maximizing the flicker filtering efficiency is also determined.

This power factor is proposed to be maintained constant during the normal operation of the device. This way, the magnitude of active and reactive power exchanged by the device become proportional to it, and also from the magnitude of the distortion in the measured signal utilized for flicker weighting. At the end, the optimal sizing of the device is that needed to keep grid flicker levels within admissible levels.

\section{STUdY CASE: FLICKER FILTERING IN MV GRIDS WITH DISTRIBUTED GENERATION. ACTIVE FILTER LOCATION AND SIZING}

This section aims to address the location and the sizing of the active flicker filter. The particular case of a MV weak network with distributed energy resources is selected. The weakness of the network (in terms of low short-circuit impedance) and the stochastic nature of the generation foresee the presence of flicker phenomena and thus motivate the installation of an active filter.

\section{A. Step 1. Network modeling}

The selected network is adopted and adapted from [13]. This document offers the progresses of a CIGRE task force in proposing a MV benchmark system for integration of renewable and distributed energy resources. This way, it seems very convenient for the purposes of the present paper. The adopted and adapted benchmark network consists in 11 buses with distributed photovoltaics and wind turbines. There are also several loads with different time variant power consumption profiles. The link between the network and the rest of the high voltage power system is represented by a synchronous generator rated at $4 \mathrm{MVA}$. The peak power generation capacity of the photovoltaics spread throughout the network is 315 $\mathrm{kW}$ and for the installed wind turbines 4.5 MW. Network topology can be observed in Figure 2. The offered numerical results are for a load flow calculation for informative purposes. Parameters for modeling lines, power transformers, as well as the active and reactive power consumption profiles for the loads of the system can be consulted in [13]. The sampling rate for load profiles is $10 \mathrm{~min}^{-1}(0.0016 \mathrm{~Hz})$, no more precise data were available for authors at the time of writing this paper. This low sampling rate implies that for the purposes of the paper, consumers are not contributing to flicker emission. This assumption is considered acceptable for the purposes of the paper, since it does not invalidate the proposed procedure for the assessment of the location and sizing of the active flicker filter.

The parameters for modeling the synchronous generator are offered in the Appendix. The model of the synchronous generator also includes a speed governor and excitation so as to control the frequency of the network and the voltage at its connection point. The speed governor corresponds to the IEEEG1 type turbine-governor model (1981) [14], and the exciter is the IEEE type DC1A [15]. Applied control parameters can be found also in the indicated bibliographic references.

Wind turbines are modeled as current sources. Their behavior is determined by modeling both the aerodynamics and the mechanics of the system as in [16]. Fast electromagnetic transients in the generator and the harmonics introduced by the power converters are omitted since they are not relevant for the purposes of the present paper (flicker phenomena is mostly experienced in sub-synchronous frequencies). To represent the variability of the power generated in a frequency range associated to flicker, the so-called effective wind speed model is adopted [17]. It permits to represent both stochastic and deterministic factors affecting the wind speed experienced by the blades of the turbine while in their rotation. Among the stochastics, the wind model includes the turbulence and the rotating sampling effect. This last effect is directly associated to flicker. The medium and long variability in wind speed (in the range of minutes up to hours) is given by adding a timevarying average wind speed to the model, and this is obtained from NREL's wind speed database [18]. The effective wind speed model provides with a wind speed time varying profile with $1 \mathrm{kHz}$ sampling rate.

Finally, the photovoltaics are also modeled as current sources, and the current-voltage equations are described in [19]. To also represent the variability of the power generated in a wide frequency spectrum, real solar irradiance data at 10 $\mathrm{Hz}$ sampling rate are adopted from NREL's database [20] and for different days (data with higher sampling rate were not found at the time of writing the paper).

\section{B. Step 2. Definition of simulation scenarios}

To evaluate flicker phenomena for the location of the active filter, $N$ network scenarios are considered concerning different renewable generation and load levels (see Table II).

TABLE II. SIMULATION SCENARIOS FOR FLICKER ASSESSMENT

\begin{tabular}{ccc}
\hline No. & Load & Wind \& PV \\
\hline 1 & average & average \\
2 & low & average \\
3 & high & average \\
4 & average & low \\
5 & average & high \\
6 & high & high \\
\hline
\end{tabular}

Without considering any filtering, the flicker levels throughout the network are presented in Table III and for each of the six above presented scenarios. As can be noted, the worst case scenario in terms of flicker emission is the number 6 , so the subsequent analyses for active filter location and sizing will be carried out under the conditions of this scenario. Also, it is interesting to note that the bus number 7 is the most affected by flicker phenomena.

\section{Steps 3 and 4. Assessment of flicker levels and location for the active filter}

The fluctuating voltage components to be filtered by the ideal active filter device for flicker mitigation are computed online, through the application of a high-pass second order Butterworth type filter with a cutoff frequency $0.1 \mathrm{~Hz}$. 




Fig. 2. Network topology. Numerical results are for a load flow calculation for informative purposes. Results refer to the active (MW) and reactive (Mvar) power at the terminals of each element of the network. Per unit voltage is also indicated in each bus.

TABLE III. MAXIMUM SHORT AND LONG FLICKER LEVELS FOR EACH OF THE BUSES OF THE NETWORK AND SCENARIO

\begin{tabular}{|c|c|c|c|c|c|c|c|c|c|c|c|c|}
\hline \multirow[t]{2}{*}{ Bus No. } & \multicolumn{2}{|c|}{ Scenario 1} & \multicolumn{2}{|c|}{ Scenario 2} & \multicolumn{2}{|c|}{ Scenario 3} & \multicolumn{2}{|c|}{ Scenario 4} & \multicolumn{2}{|c|}{ Scenario 5} & \multicolumn{2}{|c|}{ Scenario 6} \\
\hline & $P_{s t \_m a x}$ & $P_{l t \_\max }$ & $P_{s t \_\max }$ & $P_{l t \_\max }$ & $P_{s t \_m a x}$ & $P_{l t \_\max }$ & $P_{s t \_m a x}$ & $P_{l t \_\max }$ & $P_{s t_{-} \max }$ & $P_{l t_{-} \max }$ & $P_{s t \_\max }$ & $P_{l t_{-} \max }$ \\
\hline 2 & 0.2064 & 0.1897 & 0.1836 & 0.1693 & 0.2311 & 0.2115 & 0.1748 & 0.1607 & 0.2254 & 0.2067 & 0.2538 & 0.2316 \\
\hline 3 & 0.2641 & 0.2440 & 0.2415 & 0.2240 & 0.2881 & 0.2655 & 0.2156 & 0.1999 & 0.3006 & 0.2787 & 0.3280 & 0.3030 \\
\hline 4 & 0.2697 & 0.2494 & 0.2470 & 0.2294 & 0.2937 & 0.2708 & 0.2196 & 0.2037 & 0.3083 & 0.2859 & 0.3356 & 0.3102 \\
\hline 5 & 0.2734 & 0.2530 & 0.2509 & 0.2330 & 0.2974 & 0.2744 & 0.2223 & 0.2063 & 0.3135 & 0.2908 & 0.3408 & 0.3150 \\
\hline 6 & 0.2745 & 0.2540 & 0.2520 & 0.2340 & 0.2984 & 0.2753 & 0.2231 & 0.2070 & 0.3149 & 0.2920 & 0.3423 & 0.3163 \\
\hline 7 & 0.2750 & 0.2544 & 0.2525 & 0.2344 & 0.2989 & 0.2757 & 0.2235 & 0.2073 & 0.3156 & 0.2926 & 0.3429 & 0.3168 \\
\hline 8 & 0.2691 & 0.2488 & 0.2464 & 0.2288 & 0.2931 & 0.2702 & 0.2192 & 0.2033 & 0.3074 & 0.2851 & 0.3348 & 0.3094 \\
\hline 9 & 0.2692 & 0.2489 & 0.2465 & 0.2289 & 0.2932 & 0.2703 & 0.2193 & 0.2033 & 0.3075 & 0.2852 & 0.3349 & 0.3095 \\
\hline 10 & 0.2694 & 0.2491 & 0.2468 & 0.2291 & 0.2934 & 0.2705 & 0.2194 & 0.2035 & 0.3079 & 0.2855 & 0.3352 & 0.3098 \\
\hline 11 & 0.2695 & 0.2492 & 0.2469 & 0.2292 & 0.2935 & 0.2707 & 0.2195 & 0.2036 & 0.3080 & 0.2857 & 0.3354 & 0.3100 \\
\hline
\end{tabular}

The output of the high-pass filter serves as the control setpoint for an ideal active flicker filter device. At this point of development, this ideal device is considered as a controllable current source, with instantaneous time response and unlimited energy capacity. However, the output power is limited at 2 $\mathrm{kVA}$. This is because, at this design stage, the objective is to identify the node with the highest sensitivity to flicker mitigation filter actions. The device is operated so that it exchanges active and reactive power proportionally to the output of the above mentioned high-pass voltage filter, i.e. the voltage distortion. So at the end, any voltage distortion detected by the high-pass voltage filter, is translated into an instantaneous, proportional and saturated response in terms of active and reactive currents entering or flowing from the active filter device.

Doing this, the optimal location for the active filter can be found as that with the highest sensitivity to flicker filtering actions, i.e. that for which the minimum of the optimization objective function is achieved (see equation (1)).

It is convenient to remark at this point of development, that the action performed by the active flicker filter may be more effective while exchanging active power than while contributing with reactive power regulation and vice-versa. The discussion around whether the contribution by the active filter should be a concurrent combination of both active and reactive 
current exchanged with the grid for performance evaluation is presented in the step 5 of the methodology (see Section IV-D). Here, addressing the optimal location, the contribution of the ideal flicker filter at each of the buses of the grid is evaluated twice: one test considering that the device exchanges just active currents, and a second test considering the regulation of just reactive currents.

These analyses are carried out under the conditions of the most critical operational scenario amongst the presented in Table II, scenario number 6 , and the simulation period is 1 hour. Simulation results are summarized in Table IV. For each possible location for the active filter $i^{*}$ and scenario $j=6$, the resultant index $P_{\text {st_max }}\left(i^{*}, i, j\right)$ is indicated, while considering that the ideal active filter is exchanging just active or reactive power. Results are presented for network bus number $i=7$, as this is the bus presenting the maximum flicker emission of the entire network, regardless the corrective actions performed by the filter device (see Table III).

TABLE IV. ASSESSMENT OF THE OPTIMAL LOCATION FOR THE ACTIVE FILTER

\begin{tabular}{ccc}
\hline Loc. $i^{*}$ & $P_{s t_{-} \max }\left(i^{*}, 7,6\right), \mathrm{P}$ reg. & $P_{s t_{-} \max }\left(i^{*}, 7,6\right), \mathrm{Q}$ reg. \\
\hline 2 & 0.3376 & 0.3326 \\
3 & 0.3357 & 0.3307 \\
4 & 0.3356 & 0.3306 \\
5 & 0.3355 & 0.3305 \\
6 & 0.3353 & 0.3303 \\
7 & $\mathbf{0 . 3 3 5 3}$ & $\mathbf{0 . 3 3 0 2}$ \\
8 & 0.3355 & 0.3305 \\
9 & 0.3356 & 0.3305 \\
10 & 0.3356 & 0.3306 \\
11 & 0.3356 & 0.3306 \\
\hline
\end{tabular}

As noted in Table IV, the bus minimizing the maximum short term flicker index for the network $P_{s t \max }\left(i^{*}, i, j\right)$ is precisely the bus number 7 . This bus is thus that with maximum sensitivity to flicker filtering actions so it is the optimal placement for the active filter device. It is worth mentioning that bus number 7 holds a wind turbine, so an important flicker source. Further, it is interesting to note that in general, and comparing the second and third columns of Table IV, maximum flicker index is reduced in a greater extent while regulating reactive power than while performing active power regulation.

\section{Step 5. Optimal power factor for the active filter device}

In the previous step of the design methodology, it was demonstrated that reactive power regulation resulted more efficient than active power regulation for flicker filtering, under the conditions of the proposed network and scenario. However, the filtering effectiveness of the device can be improved by performing a control action combining both active and reactive power contribution. Active power filtering regulation gains relevance with the resistive characteristic of distribution lines. This way, filtering with active power results efficient in low voltage weak distributions, because of their noticeable resistive characteristic, but it is inefficient for filtering in high voltage networks, because they are highly inductive [8]. To find the optimal power factor to be performed by the active filter for maximizing filtering effectiveness, a set of simulations under the conditions of the scenario number 6 is conducted. In these simulations, the power factor $\cos \phi$ developed by the ideal active flicker filter is varied from 1 to 0 (so from 0 to 90 degrees). Results are plotted in Figure 3. As can be seen, the impact in flicker filtering is maximized by considering a power factor of about $\cos \phi=0.5$, so for $\phi=60$ degrees.

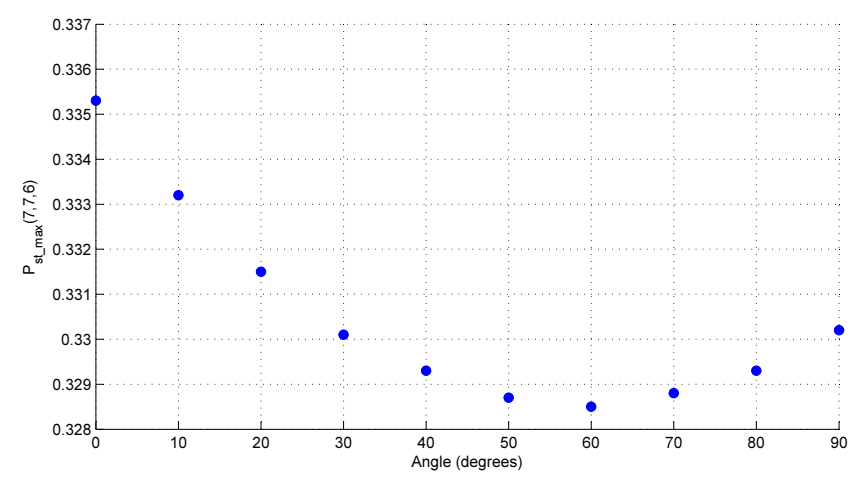

Fig. 3. Optimal power factor for the active filter.

E. Step 6. Determination of the required power and energy capacity for the active filter

From the conclusions achieved in the previous design step, the active filter is viewed as an ideal current source exchanging both active and reactive currents, and so far the power capacity is limited so as to configure a very small device.

Now, this limitation in the power capacity is removed. Thus, and in order to find the optimal sizing, filtering actions are progressively increased in magnitude till lowering flicker levels down to admissible ones. For the sake of clarity, Figure 4 presents the conceptual operational strategy of the active filter, including the weighting factor $K$. As can be noted, the input signal, i.e. the network voltage, is passed through a high pass filter, which derives the fluctuating components to be smoothed out for flicker reduction. This signal is weighted by a factor $K$, yielding the magnitude of the current to be exchanged with the grid. Then, the resultant is affected by the optimal power factor to be developed, finally yielding the active and reactive current set-points for the active filter.

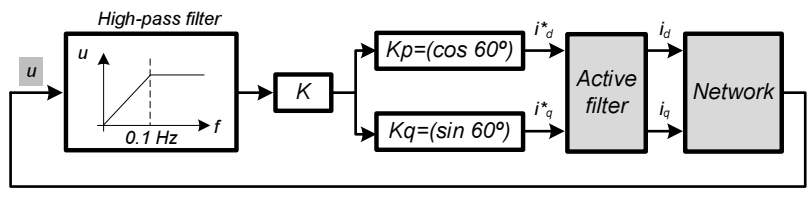

Fig. 4. Conceptual control system for the active filter.

Table $\mathrm{V}$ summarizes the sizing process, presenting the obtained maximum short and long term flicker indices while considering different values for parameter $K$. As a reminder, values refer to bus number 7 and the worst case network scenario (number 6).

As can be noted, the maximum short term flicker indices are kept within admissible levels (see Table I) for all considered values for parameter $K$. However, the maximum registered long term flicker indices do not fit with the normative (see Table I) until considering values for parameter $K$ equal or greater than 0.6, thus yielding the minimum size for the active 
TABLE V. SIZING ASSESSMENT FOR THE ACTIVE FILTER

\begin{tabular}{ccc}
\hline$K$ & $P_{\text {st_ } \max }(7,7,6)$ & $P_{l t_{-} \max }(7,7,6)$ \\
\hline 0.3 & 0.2956 & 0.2736 \\
0.4 & 0.2825 & 0.2615 \\
0.5 & 0.2702 & 0.2503 \\
$\mathbf{0 . 6}$ & $\mathbf{0 . 2 5 9 2}$ & $\mathbf{0 . 2 4 0 0}$ \\
$\mathbf{0 . 9}$ & $\mathbf{0 . 2 2 9 8}$ & $\mathbf{0 . 2 1 3 0}$ \\
1.5 & 0.1869 & 0.1738 \\
2.4 & 0.1454 & 0.1356 \\
\hline
\end{tabular}

filter. Oversizing the system for future network modifications, parameter $K=0.9$ is selected for design. Applying this parameter, and from simulation results, the achieved ratings for the active filter are presented in Table VI.

TABLE VI. RATINGS FOR THE ACTIVE FILTER, $K=0.9$

\begin{tabular}{lc}
\hline Power capacity & $80 \mathrm{kVA}$ \\
Energy storage capacity & $100 \mathrm{kWs}$ \\
\hline
\end{tabular}

The obtained power and energy storage capacities along with the required short time responses and high cyclability for flicker smoothing purposes, derive short-term storage devices such as flywheels and ultracapacitors suitable for this application.

Further showing the effectiveness of the designed active filter, Figure 5 plots the spectrum of the voltage at network bus 7, with and without considering the application of the filter. As can be noted, the voltage disturbances from $0.1 \mathrm{~Hz}$ are diminished through the application of the active filter. The graph also plots the results for a quite oversized active filter rated at 1.2 MVA for comparison purposes.

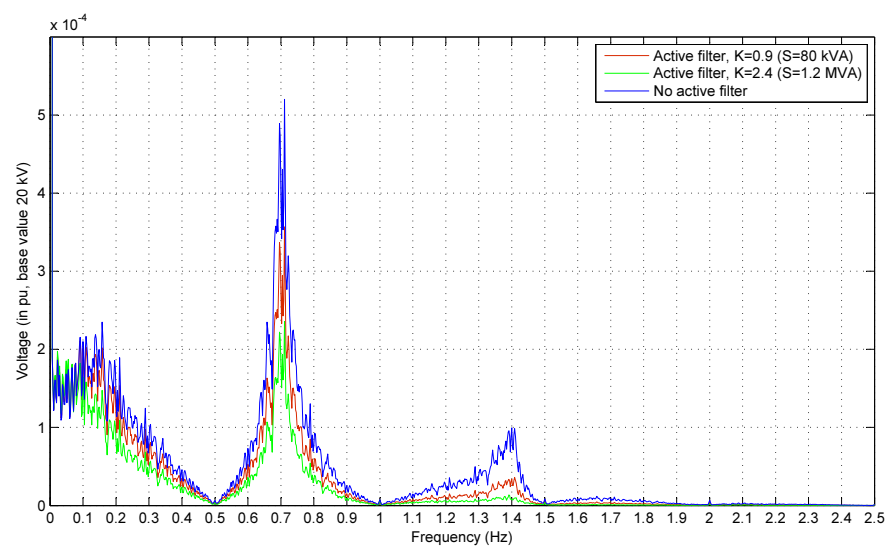

Fig. 5. Spectrum of the voltage at bus 7 with and without the contribution of the active filter.

\section{COnclusions}

This paper presented a methodology for addressing the location and sizing of an active filter for voltage flicker. The methodology was tested in the particular study case of a weak distribution network with high penetration of renewable generation. The designed system succeeded in reducing flicker levels down to admissible ones under the particular conditions of the proposed study case. Further, it is worth noting that by including energy storage capacity, the performance of the filter device was improved while compared to the case it performs flicker filtering just through reactive power regulation (see Figure 3).

\section{APPENDIX}

The parameters of the synchronous generator are extracted from DigSilent PowerFactory database [21] (in p.u. stator base): rated power $4 \mathrm{MVA}$, rated stator line-to-line voltage $11 \mathrm{kV}, r_{s}=0.0504, x_{d}=1.5, x_{q}=0.75, x_{l s}=0.1$, $x_{d}^{\prime}=0.256, T_{d}^{\prime}=0.53 \mathrm{~s}, x_{d}^{\prime \prime}=0.168, x_{q}^{\prime \prime}=0.184, T_{d}^{\prime \prime}=0.03$ $\mathrm{s}, T_{q}^{\prime \prime}=0.03 \mathrm{~s}$, inertia time constant $H^{q}=2 \mathrm{~s}$.

\section{REFERENCES}

[1] The European Commission. The European Strategy Energy Technology Plan. Available online: http://ec.europa.eu/energy/technology/set_plan/set_plan_en.htm. Access date: 12.11 .2014

[2] IEC 61000-4-15. Electromagnetic compatibility (EMC) Part 4-15: Testing and measurement techniques flickermeter functional and design specifications.

[3] ABB. Power protection. ABB Insider, Vol. 6, Issue 12. Available online: http://www05.abb.com/global/. Accessed: 12.11.2014.

[4] IEC 61400-21. Wind turbines Part 21: Measurement and assessment of power quality characteristics of grid connected wind turbines.

[5] A. Wilkins, J. Veitch, and B. Lehman, "LED lighting flicker and potential health concerns: IEEE Standard PAR1789 update," in IEEE Energy Conversion Congress and Exposition. Atlanta, GA, 2010, pp. 171-178.

[6] C. Han, A.Q. Huang, M.E. Baran, S. Bhattacharya, W. Litzenberger, L. Anderson, et al., "STATCOM impact study on the integration of large wind farm into a weak loop power system," IEEE Transactions on Energy Conversion, vol. 23, no. 1, pp. 22633, 2007.

[7] C. Abbey, G. Joos, "Supercapacitor energy storage for wind energy applications," IEEE Transactions on Industry Applications, vol. 43, no. 3, pp. 769-776, 2007.

[8] F. Girbau-Llistuella, A. Sumper, F. Díaz-González, and S. GalceranArellano, "Flicker mitigation by reactive power control in wind farm with doubly fed induction generators," International Journal of Electrical Power and Energy Systems, vol. 55, pp. 285-296, 2014.

[9] F. Díaz-González, F.D. Bianchi, A. Sumper, and O. Gomis-Bellmunt, "Control of a flywheel energy storage system for power smoothing in wind power plants," IEEE Transactions on Energy Conversion, vol. 29, no. 1, pp. 204-214, 2014

[10] J. Marcos, L. Marroyo, E. Lorenzo, D. Alvira, E. Izco, "From irradiance to output power fluctuations: the pv plant as a low pass filter," Progress in Photovoltaics: Research and Applications, vol. 19, pp. 505-510, 2010.

[11] C. Whitaker, J. Newmiller, M. Ropp, B. Norris, "Renewable systems interconnection study: distributed photovoltaic systems design and technology requirements," Sandia Report SAND2008-0946P, 2008.

[12] IEC, "IEC 61000-3-7. Electromagnetic Compatibility (EMC), Part 3: Limits Section 7: assessment of emission limits for the connection of fluctuating installations to MV, HV and EHV power systems, 2nd ed.," 2008.

[13] CIGRE, "CIGRE task force C6.04.02. Benchmark systems for network integration of renewable and distributed energy resources," 2013.

[14] Siemens, "BSOL controllers - Standard 1," 2008.

[15] IEEE, "IEEE Std. 421.5-2005. IEEE Recommended practice for excitation system models for power system stability studies," 2005.

[16] F. Díaz-González, A. Sumper, O. Gomis-Bellmunt, F.D. Bianchi, "Energy management of flywheel-based energy storage device for wind power smoothing," Applied Energy, vol. 110, pp. 207-219, 2013.

[17] F.D. Bianchi, H. de Batista, R.J. Mantz, Wind turbine control systems. Principles, modeling and gain scheduling design. Springer, 2007

[18] National Renewable Energy Laboratory webpage: http://www.nrel.gov/. Access date: 20.11.2014.

[19] M. Gradella-Villalva, J.R. Gazoli, E.R. Filho, "Comprehensive approach to modeling and simulation of photovoltaic arrays," IEEE Transactions on Power Electronics, vol. 24, no. 5, pp. 1198-1208, 2009.

[20] National Renewable Energy Laboratory. MIDC research stations. Webpage: http://www.nrel.gov/midc/research/. Access date: 20.11.2014.

[21] DIgSILENT GmbH, "DIgSILENT PowerFactory v.14, user manual," Germany, 2009 